\title{
A Specific Method for Solving Fractional Delay Differential Equation via Fraction Taylor's Series
}

\author{
Ming-Jing Du (iD \\ Institute of Computer Information Management, Inner Mongolia University of Finance and Economics, Hohhot 010070, China \\ Correspondence should be addressed to Ming-Jing Du; 724297269@qq.com
}

Received 14 July 2021; Revised 8 October 2021; Accepted 10 December 2021; Published 4 January 2022

Academic Editor: Ahmed Salem

Copyright (c) 2022 Ming-Jing Du. This is an open access article distributed under the Creative Commons Attribution License, which permits unrestricted use, distribution, and reproduction in any medium, provided the original work is properly cited.

It is well known that the appearance of the delay in the fractional delay differential equation (FDDE) makes the convergence analysis very difficult. Dealing with the problem with the traditional reproducing kernel method (RKM) is very tricky. The feature of this paper is to gain a more credible approximate solution via fractional Taylor's series (FTS). We use the FTS to deal with the delay for improving the accuracy of the approximate solutions. Compared with other methods, the five numerical examples demonstrate the accuracy and efficiency of the proposed method in this paper.

\section{Introduction}

For the past few years, FDDE have been applied in various fields of neoteric science and engineering such as economics, physics, dynamics, hydrology, finance, signal processing, neural network, and control theory [1]. There has been a growing interest in researching numerical methods for this equation. Many experts and scholars use various methods to study this kind of model, including Legendre seudospectral method [2], a new Legendre operational technique [3], the homotopy perturbation method [4], a linearized compact finite difference scheme [5], the techniques of Tau method and collocation method [6], the adaptation of a fractional backward difference method [7], extended Chebyshev wavelet method [8], and the variational iteration and Adomian decomposition method [9]. It is worth mentioning that in [10], Esra investigates a couple of different financial and economic models by market equilibrium and option pricing with three different fractional derivatives and proves the precision of the Sumudu transform and decomposition series method constructed by the Laplace transform. In [11], the Rabotnov exponential kernel is used to define the fundamental calculus of the fractional derivative and solve the nonlinear dispersive wave model. In [12], Asif uses three methods (the expansion method, the finite difference method, and the Laplace perturbation method) to solve the time-fractional nonlinear BurgerFisher equation. In [13], Laiq uses the optimal auxiliary function method for solving the general partial differential equations. In [14], Abdulnasir adopts a new simple and effective new algorithm based on one of the Appell polynomials, namely, Genocchi polynomials, to solve the generalized Pantograph equations, the FDDE with neutral terms, and delay differential system with constant and variable coefficients. In [15], Phang uses an operational matrix method for solving the FDDEs. In [16], Salem gives the existence and uniqueness of the coupled system of nonlinear fractional Langevin equations (FLE) with multipoint and nonlocal integral boundary conditions (NIBCs). In [17], Salem studies the existence and uniqueness of the solution for the same class of the equation in a slightly different form with antiperiodic and NIBCs. In [18], Selam and Dosari conduct research on the existence results of solutions for boundary value problems of inclusion type. In [19], Salem and Alghamdi give the existence and uniqueness of solutions for the Langevin equation (LE) that has Caputo fractional derivatives of two different orders. In [20], Salem et al. give the existence results for an infinite system of LEs involving generalized derivatives of two distinct fractional orders with three-point boundary conditions. 
In this study, we focus on a more general case in math, considering the following FDDE arising from economic models.

$$
\left\{\begin{array}{l}
D^{v} u(t)=a(t) u(t-\tau)+b(t) u^{\prime}(t-\sigma)+c(t) u(t)+f(t), \quad t \in[0, T] \\
u(t)=\varphi(t), \quad t \in[-\tau, 0] \cup[-\sigma, 0]
\end{array}\right.
$$

$\tau$ and $\sigma$ are the constant delays. $a(t), b(t), c(t), \varphi(t)$, and $f(t)$ are the continuous functions. $D^{\nu}$ is the Riemann-Liouville fractional derivative of order $v>0$.

This paper is organized as follows: in Section 2, some important definitions about the fractional Taylor's series, reproducing kernels, and their spaces are reviewed. The process of solving problem (1) is presented in Section 3. The five numerical examples are given in Section 4. Then, we give the conclusion in Section 5.

\section{Fractional Taylor's Series}

Definition 1. The $R-L$ fractional derivative of order $\alpha$ is defined as [1]

$$
D^{\alpha} u(x)=\frac{1}{\Gamma(n-\alpha)} \frac{\mathrm{d}^{n}}{\mathrm{~d} x^{n}} \int_{0}^{x} \frac{u(t)}{(x-t)^{n-\alpha}} \mathrm{d} t
$$

where $\Gamma(\cdot)$ is the gamma function and $n=[\alpha]+1,[a]$ denotes the greatest integer smaller than $\alpha$.

Lemma 1. Let us suppose that $m<\alpha \leq m+1, m \in N-\{0\}$ and that $u(t)$ has derivatives of order $k$ (integer), $1 \leq k \leq m$. Let us suppose further that $u^{(m)}(t)$ has a FTS of order $\alpha-m=$ $: \beta$ provided by the expression (the symbol = means that the left side is defined by the right one) [21]:

$$
\begin{array}{r}
u^{(m)}(t+h)=\sum_{k=0}^{\infty} \frac{h^{k(\alpha-m)}}{\Gamma[1+k(\alpha-m)]} D^{k(\alpha-m)} u^{(m)}(t), \\
m<\alpha \leq m+1 .
\end{array}
$$

Next, on integrating this series with respect to $h$ yields

$$
u(t+h)=\sum_{k=0}^{m} \frac{h^{k}}{k !} u^{(k)}(t)+\sum_{k=1}^{\infty} \frac{h^{(k \beta+m)}}{\Gamma[k \beta+m+1]} u^{(k \beta+m)}(t), \quad \beta:=\alpha-m
$$

Definition 2. $W_{2}^{n+1}[0, T]=\left\{u(t) \mid u(t), \ldots, u^{(n)}(t)\right.$ is onevariable absolutely continuous function $\left.(\mathrm{ACF}), u^{(n+1)}(t) \in L^{2}[0, T]\right\}$. The inner product is given by

$$
\begin{aligned}
\langle u(t), w(t)\rangle_{W_{2}^{n+1}}= & \sum_{i=0}^{n} u^{(i)}(0) w^{(i)}(0) \\
& +\int_{0}^{T} u^{(n+1)}(t) w^{(n+1)}(t) \mathrm{d} t .
\end{aligned}
$$

Lemma 2. The space $W_{2}^{n+1}[0, T]$ is a reproducing kernel space (RKS) and its reproducing kernel $(R K) R_{t}^{n+1}(y)$ is denoted by [12]

$$
R_{t}^{m+1}(y)= \begin{cases}\sum_{i=0}^{n} t^{i} y^{i}+\int_{0}^{t}(t-x)^{n}(y-x)^{n} \mathrm{~d} x, & t<y, \\ \sum_{i=0}^{n} t^{i} y^{i}+\int_{0}^{y}(t-x)^{n}(y-x)^{n} \mathrm{~d} t, & y<t .\end{cases}
$$

\section{Solution of the Equation}

By Lemma 1, $f(t)$ can be expressed via fractional Taylor's series.

$$
f(t)=D^{\nu} u(t)-a(t) \cdot\left[\sum_{k=0}^{1} \frac{(-\tau)^{k}}{k !} u^{(k)}(t)+\frac{(-\tau)^{(\beta+1)}}{\Gamma[\beta+2]} u^{(\beta+1)}(t)\right]-b(t) \cdot \sum_{k=0}^{1} \frac{(-\sigma)^{k(\alpha-1)}}{\Gamma[1+k(\alpha-1)]} D^{k(a-1)} u^{\prime}(t)-c(t) u(t) .
$$

Using TRKM, we need to define a bounded linear operator $L[22]: W_{2}^{3}[0, T] \longrightarrow W_{2}^{1}[0, T]$, let

$$
(L u)(t)=f(t)
$$

so the solutions of (1) is the solution of (8). $R_{x}^{3}(y)$ [14] is the RK of the RKS $W_{2}^{3}[0, T] ; R_{x}^{1}(y)$ is the RK of the RKS $W_{2}^{1}[0, T]$. According to [15], the exact solution of $(7)$ is expanded by $u(t)=\sum_{j=1}^{\infty} f\left(t_{j} \zeta_{j}(t)\right)$. The approximate 
solution of (7) is expanded by $u_{N}(t)=\sum_{j=1}^{N} f\left(t_{j}\right) \zeta_{j}(t)$. For the convergence theorem, please refer to [23]. Error estimate is as follows.

Theorem 1. $\forall t \in[0, T], \exists H=\left\|u(t)-u_{N}(t)\right\| \cdot \| \partial R(\eta, t) /$ $\left.\partial t\right|_{t=\theta} \|$; there is always $\left\{\left(t_{j}\right)\right\}_{j=1}^{\infty}$ satisfying $t-t_{j}=1 / N$, so $\left|u(t)-u_{N}(t)\right| \leq H / N$.
Proof Due to $L u\left(t_{j}\right)=L u_{N}\left(t_{j}\right)$, then

$\left|L u(t)-L u_{N}(t)\right|=\left|L u(t)-L u\left(t_{j}\right)-\left[L u_{N}(t)-L u_{N}\left(t_{j}\right)\right]\right|$. then

Due to $u(t)=\langle u(\eta), R(\eta, t)\rangle, L u(t)=\langle u(\eta), L R(\eta, t)\rangle$,

$$
\begin{aligned}
& \text { So, } \\
& \qquad\left|u(t)-u_{N}(t)\right|=\left|L^{-1}\left[L u(t)-L u_{N}(t)\right]\right| \\
& \leq\left|\left\langle u(\eta)-u_{N}(\eta), L^{-1} L R(\eta, t)-L^{-1} L R\left(\eta, t_{j}\right)\right\rangle\right| \\
& \leq\left\|u-u_{N}\right\| \cdot\left\|R(\eta, t)-R\left(\eta, t_{j}\right)\right\| .
\end{aligned}
$$$$
L u(t)-\operatorname{LT}_{N}(t)=L u(t)-L u\left(t_{j}\right)-\left[L u_{N}(t)-L u_{N}\left(t_{j}\right)\right]
$$$$
=\left\langle u(\eta), \operatorname{LR}(\eta, t)-\operatorname{LR}\left(\eta, t_{j}\right)\right\rangle-\left\langle u_{N}(\eta), \operatorname{LR}(\eta, t)-\operatorname{LR}\left(\eta, t_{j}\right)\right\rangle
$$$$
=\left\langle u(\eta)-u_{N}(\eta), \operatorname{LR}(\eta, t)-\operatorname{LR}\left(\eta, t_{j}\right)\right\rangle .
$$

Because we take the norm of $\left\|R(\eta, t)-R\left(\eta, t_{j}\right)\right\|$, for $\forall \eta$, the RK function $R(\eta, t)$ is derived on $t$ in the interval of $[0, T]$; therefore, $R(\eta, t)-R\left(\eta, t_{j}\right)=\partial R(\eta, t) /\left.\partial t\right|_{t=\theta^{*}}\left(t-t_{j}\right)$, let $H=\left\|u(t)-u_{N}(t)\right\| \cdot\left\|\partial R(\eta, t) /\left.\partial t\right|_{t=\theta}\right\|$, hence

$$
\left|u(t)-u_{N}(t)\right| \leq\left\|u(t)-u_{N}(t)\right\| \cdot\left\|\left.\frac{\partial R(\eta, t)}{\partial t}\right|_{t=\theta} \cdot\left(t-t_{j}\right)\right\|=\frac{H}{N} .
$$

$$
\left\{\begin{array}{l}
D^{v} u(t)=-u^{\prime}(t-1)-u(t)+f(t), \quad t>0, \\
u(t)=t^{2} \sin (\pi t), \quad t \in[-1,0],
\end{array}\right.
$$

with $v=3 / 2, f(t)=(t-1) \cdot[-\pi(t-1)(\cos \pi t-2 \sin \pi t]$ $+t^{2} \sin (\pi t)+D^{3 / 2} t^{2} \sin (\pi t)$ which is compatible with the exact solution $u(t)=t^{2} \sin (\pi t)$. We estimated the parameter $m=1, k=1, \alpha=1.5$, by Mathematical 7.0, $N=14$; Figure 1 shows the absolute errors by two methods, the left one is given by the traditional RKM, and the right one is given by the proposed method in this paper. It is obvious that the proposed method in this paper is more accurate than the traditional RKM.

Example 2. Consider the following delay integro-differential equation [24]:

\section{Numerical Examples}

Example 1. Consider the following FDDE:

$$
\left\{\begin{array}{l}
-t u^{\nu}(t)+t u^{\prime}(t)+u(t)+t u^{\prime}(t-1)+3 u(t+1)=f(t)+\int_{-1}^{1} t u^{2}(x-1) \mathrm{d} x \\
u(0)=u^{\prime}(0)=0
\end{array}\right.
$$

with $v=2, f(t)=23 / 48-13 \cos 4 / 16+(t-1) t \cos (t-1)$ $+(t-2) t \cos t-3 \sin 4 / 8-t \sin (1-t)+(2+t) t \sin t+3$ $(1+t) \sin (1+t)$; the exact solution of (14) is $u(t)=t \sin (t)$. The delay term in the equation is expanded by fractional Taylor's series. We estimated the parameters $m=1, k=1$, $\alpha=1.1$, and $\beta=0.1$. By Mathematical 7.0, $N=10$, Figure 2 presents the absolute errors by two methods, the left one is given by the traditional RKM, and the right one is given by the proposed method in this paper. It is obvious that the proposed method in this paper is more precise than the traditional RKM.
Example 3. Consider the following FDDE:

$$
\left\{\begin{array}{l}
u^{v}(t-1)-t u(t-1)=\frac{1}{8}\left(3-5 t+3 t^{3}-t^{4}\right) \\
u(0)=0
\end{array}\right.
$$

with $v=1$, the exact solution $u(t)=(t / 2)^{3}$. The delay term in the equation is expanded by fractional Taylor's series. We estimated the parameters $m=1, k=1, \alpha=1.5$, and $\beta=0.5$. By Mathematical 7.0, $N=12$, Figure 3 shows the absolute errors by two methods, the left one is given by the traditional 

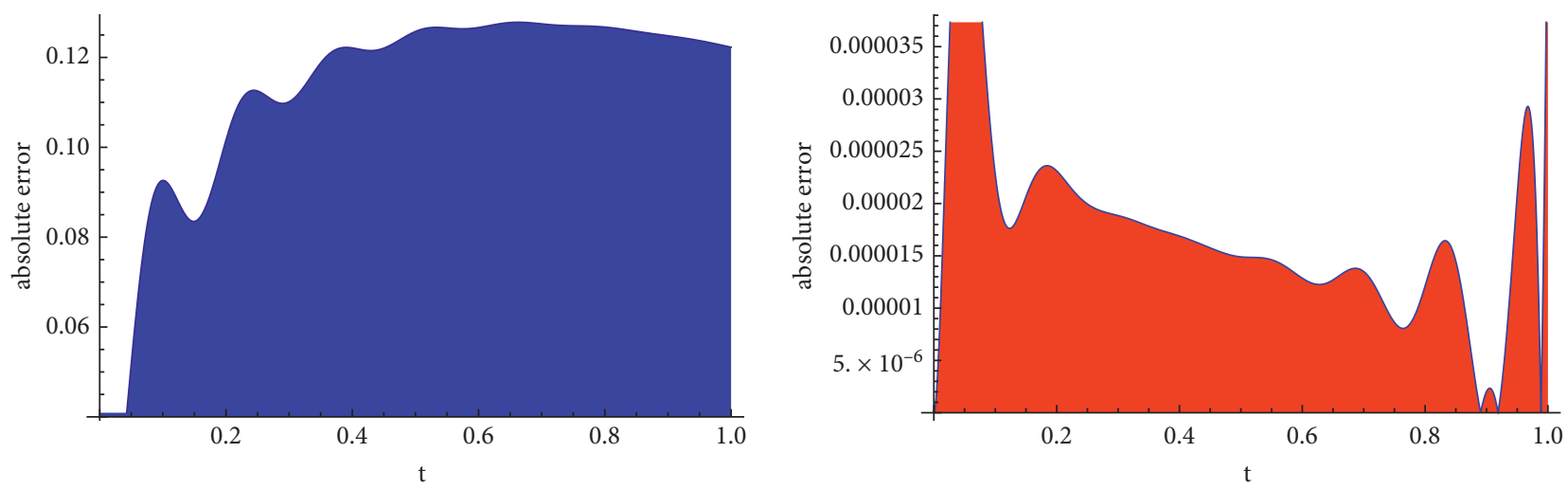

FIgURE 1: Absolute errors of Example 1 by the traditional RKM (left) and the proposed method in this paper (right, $m=1, k=1, \alpha=1.5)$.
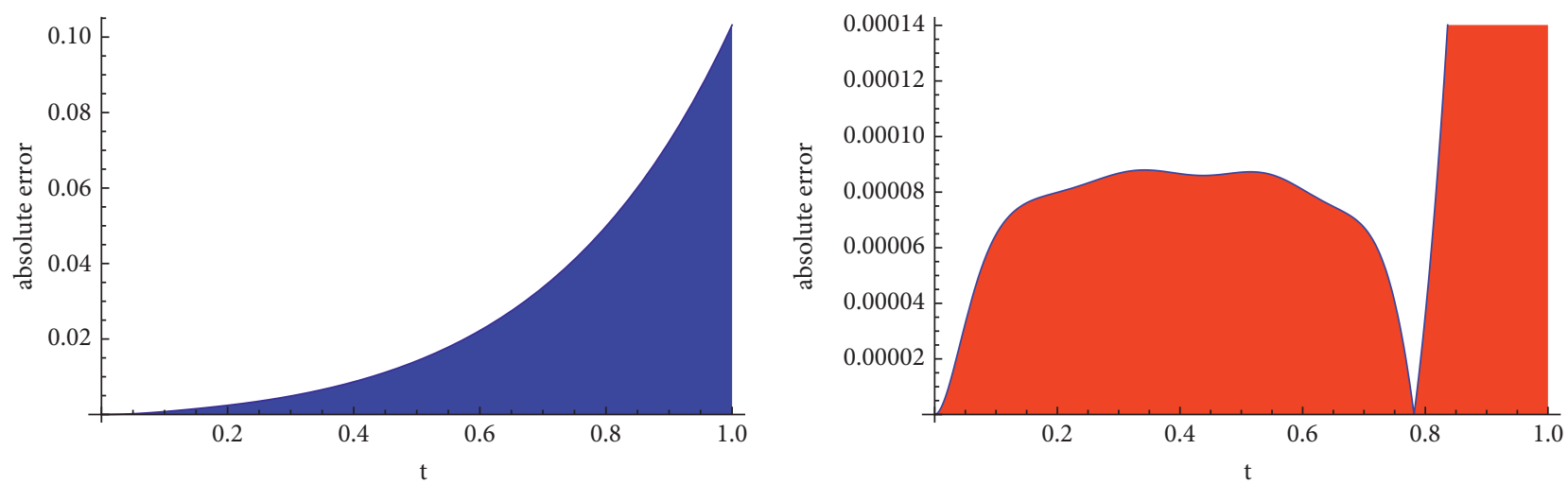

FIGURE 2: Absolute errors of Example 2 by the traditional RKM (left) and the proposed method in this paper (right, $m=1, k=1, \alpha=1.1, \beta=0.1$ ).
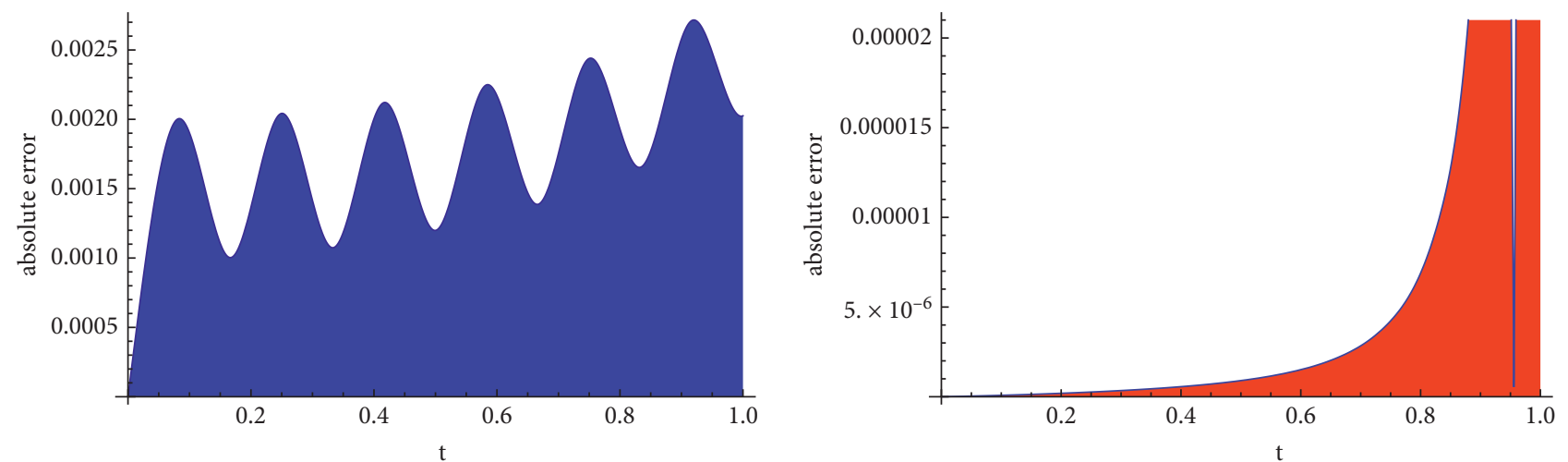

FIGURE 3: Absolute errors of Example 3 by the traditional RKM (left) and the proposed method in this paper (right, $m=1, k=1, \alpha=1.5, \beta=0.5$ ).

RKM, and the right one is given by the proposed method in this paper. It is obvious that the proposed method in this Example 4. Consider the following FDDE [25]: paper is more precise than the traditional RKM.

$$
\left\{\begin{array}{l}
D^{v} u(t)+u(t)-u(t-\tau)=\frac{2}{\Gamma(3-v)} t^{2-\nu}-\frac{1}{\Gamma(2-\nu)} t^{1-\nu}+2 \tau t-\tau^{2}-\tau \\
u(0)=0
\end{array}\right.
$$




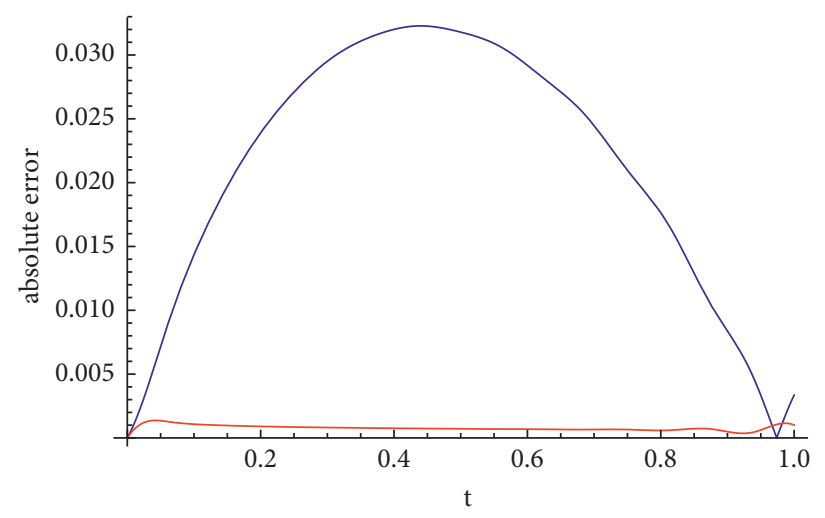

Figure 4: Absolute errors of Example 4 by traditional method (blue line) and the proposed method in this paper (red line, $m=1, k=1$, $\alpha=1.5, \beta=0.5)$.

TABle 1: Numerical solution comparison of Example 5 by the five methods.

\begin{tabular}{ccccccc}
\hline$x_{i}$ & Exact solution & ADM [26] & Method [25] & Hermite wavelet [27] & Traditional RKM & The proposed method \\
\hline 0.0 & 1.0000 & 1.0000 & 1.0000 & 1.0000 & 1.0000 & 1.0000 \\
0.2 & 0.8187 & 0.8187 & 0.8187 & 0.8187 & 0.8189 & 0.8187 \\
0.4 & 0.6703 & 0.6703 & 0.6703 & 0.6703 & 0.6718 & 0.6703 \\
0.6 & 0.5488 & 0.5488 & 0.5488 & 0.5488 & 0.5529 & 0.5488 \\
0.8 & 0.4493 & 0.4493 & 0.4494 & 0.4493 & 0.4575 & 0.4493 \\
\hline
\end{tabular}

We estimated the parameters $v=0.2, \tau=0.1, m=1$, $k=1, \alpha=1.5$, and $\beta=0.5$. The exact solution of (16) is $u(t)=t^{2}-t$. By Mathematical 7.0, $N=16$, Figure 4 presents the absolute errors by two methods. The left one is given by the traditional RKM and the right one is given by the proposed method in this paper. It is obvious that the proposed method in this paper is more precise than the traditional RKM.

Example 5. Consider the following FDDE [25-27]:

$$
\left\{\begin{array}{l}
D^{\nu} u(t)=-u(t)-u(t-\tau)+e^{-t+\tau} \\
u(0)=1, u^{\prime}(0)=-1, u^{\prime \prime}(0)=1
\end{array}\right.
$$

The exact solution is $u(t)=e^{-t}$ when $v=3$. We estimated the parameters $\tau=0.3, m=1, k=1, \alpha=1.5$, and $\beta=0.5$. By Mathematical 7.0, $N=12$, Table 1 shows the numerical comparison by the five methods.

\section{Conclusion}

In this study, the FTS and the RKs are successfully used to solve a class of PDDEs. The comparison between the proposed method and other methods is given in Section 4, which shows that the proposed method in this paper is a very satisfactory numerical method. In addition, we can also use this new method to solve the option pricing model.

\section{Data Availability}

The data used to support the findings of this study are available from the corresponding author upon request.

\section{Conflicts of Interest}

The author declares no conflicts of interest.

\section{Acknowledgments}

This paper has been supported by the Natural Science Foundation of Inner Mongolia (No. 2019BS01011), the Research Program of Science and Technology at Universities of Inner Mongolia Autonomous Region (No. NJZY20158), and the Program for Young Talents of Science and Technology in Universities of Inner Mongolia Autonomous Region (No. NJYT-20-B18).

\section{References}

[1] M. Xu and Y. Lin, "Simplified reproducing kernel method for fractional differential equations with delay," Applied Mathematics Letters, vol. 52, pp. 156-161, 2016.

[2] N. Qiu, "Application of time fractional delay differencential equations in fluid dynamics," J. Shenyang. Univ.vol. 28, pp. 170-172, 2016.

[3] A. H. Bhrawy and S. S. E. Eldien, "A new Legendre operational technique for delay fractional optimal control problems," Calcolo, vol. 53, no. 4, pp. 521-543, 2016.

[4] M. G. Uludag and F. Erdogan, "Numerical solution of timefractional nonlinear PDEs with proportional delays by homotopy perturbation method," Applied Mathematical Modelling, vol. 40, no. 13-14, pp. 6639-6649, 2016.

[5] Q. Zhang, M. Ran, and D. Xu, "Analysis of the compact difference scheme for the semilinear fractional partial differential equation with time delay," Applicable Analysis, vol. 96, no. 11, pp. 1867-1884, 2017. 
[6] A. Saadatmandi and M. Dehghan, "A new operational matrix for solving fractional-order differential equations," Computers \& Mathematics with Applications, vol. 59, no. 3, pp. 1326-1336, 2010.

[7] M. L. Morgado, N. J. Ford, and P. M. Lima, "Analysis and numerical methods for fractional differential equations with delay," Journal of Computational and Applied Mathematics, vol. 252, pp. 159-168, 2013.

[8] U. Saeed, M. U. Rehman, and M. A. Iqbal, "Modified Chebyshev wavelet methods for fractional delay-type equations," Applied Mathematics and Computation, vol. 264, pp. 431-442, 2015.

[9] M. Dehghan and R. Salehi, "Solution of a nonlinear time-delay model in biology via semi-analytical approaches," Computer Physics Communications, vol. 181, no. 7, pp. 1255-1265, 2010.

[10] A. Karatas, A. Ali, and Y. Mehmet, "New illustrative applications of integral transforms to financial models with fifferent fractional derivatives," Chaos, Solitons \& Fractals, vol. 146, Article ID 110877, 2021.

[11] M. Yavuz and N. Sene, "Fundamental calculus of the fractional derivative defined with Rabotnov exponential kernel and application to nonlinear dispersive wave model," Journal of Ocean Engineering and Science, vol. 6, no. 2, pp. 196-205, 2021.

[12] A. Yokus and M. Yavuz, "Novel comparison of numerical and analytical methods for fractional burger-Fisher equation," Discrete \& Continuous Dynamical Systems - S, vol. 14, no. 7, pp. 2591-2606, 2021.

[13] L. Zada, R. Nawaz, K. S. Nisar et al., "New approximate-analytical solutions to partial differential equations via auxiliary function method," Partial Differential Equations in Applied Mathematics, vol. 4, Article ID 100045, 2021.

[14] A. Isah and C. Phang, "Operational matrix based on Genocchi polynomials for solution of delay differential equations," Ain Shams Engineering Journal, vol. 9, pp. 2123-2128, 2018.

[15] C. Phang, Y. T. Toh, and F. S. Nasrudin, "An operational matrix method based on poly-Bernoulli polynomials for solving fractional delay differential equations," Computation, vol. 8 , no. 3, 2020.

[16] A. Salem, F. Alzahrani, and M. Alngga, "Coupled system of nonlinear fractional Langevin equations with multipoint and nonlocal integral boundary conditions," Mathematical Problems in Engineering, vol. 202015 pages, Article ID 7345658, 2020.

[17] A. Salem, "Existence results of solutions for anti-periodic fractional Langevin equation," Journal of Applied Analysis \& Computation, vol. 10, no. 6, pp. 2557-2574, 2020.

[18] A. Salem and A. A. Dosari, "A countable system of fractional inclusions with periodic, almost, and antiperiodic boundary conditions," Mathematical Problems in Engineering, vol. 202110 pages, Article ID 6653106, 2021.

[19] A. Salem and B. Alghamdi, "Multi-point and anti-periodic conditions for generalized Langevin equation with two fractional orders," Fractal and Fractional, vol. 3, no. 4, 2019.

[20] A. Salem, H. M. Alshehri, and L. Almaghamsi, "Measure of noncompactness for an infinite system of fractional Langevin equation in a sequence space," Adv.Differ.Eq.vol. 2021, Article ID 033022, 2021.

[21] G. Jumarie, "From Lagrangian mechanics fractal in space to space fractal Schrödinger's equation via fractional Taylor's series," Chaos, Solitons \& Fractals, vol. 41, no. 4, pp. 1590-1604, 2009.

[22] Y. Wang, M. Du, and C. Temuer, "A modified reproducing kernel method for a time-fractional telegraph equation," Thermal Science, vol. 21, no. 4, pp. 1575-1580, 2017.
[23] Y. Wang, C. Temuer, and J. Pang, "New algorithm for secondorder boundary value problems of integro-differential equation," Journal of Computational and Applied Mathematics, vol. 229, pp. 1-6, 2009.

[24] K. Sadri, A. Amini, and C. Cheng, "A new numerical method for delay and advanced integro-differential equations," $\mathrm{Nu}$ merical Algorithms, vol. 77, no. 2, pp. 381-412, 2018.

[25] P. Rahimkhani, Y. Ordokhani, and E. Babolian, "A new operational matrix based on Bernoulli wavelets for solving fractional delay differential equations," Numerical Algorithms, vol. 74, no. 1, pp. 223-245, 2017.

[26] D. J. Evans and K. R. Raslan, "The Adomian decomposition method for solving delay differential equation," International Journal of Computer Mathematics, vol. 82, pp. 1-6, 2004.

[27] U. Saeed and M. U. Rehman, "Hermite wavelet method for fractional delay differential equations," Journal of Difference Equations, vol. 2014, Article ID 359093, 8 pages, 2014. 